Effect of Prepregnancy Pertussis Vaccination in Young Infants Peer-reviewed author version

Maertens, Kirsten; TRAN, Mai Phuong Thao; HENS, Niel; Van Damme, Pierre \& Leuridan, Elke (2017) Effect of Prepregnancy Pertussis Vaccination in Young Infants. In: JOURNAL OF INFECTIOUS DISEASES, 215(12), p. 1855-1861.

DOI: 10.1093/infdis/jix176

Handle: http://hdl.handle.net/1942/24324 


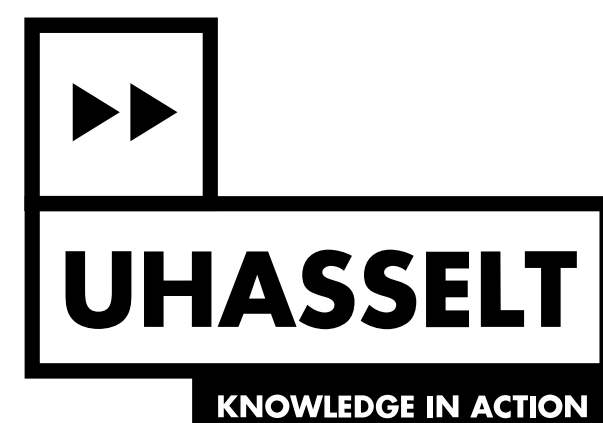

Effect of Prepregnancy Pertussis Vaccination in Young Infants Link Peer-reviewed author version

Made available by Hasselt University Library in Document Server@UHasselt

Reference (Published version):

Maertens, Kirsten; Tran, Mai Phuong Thao; Hens, Niel; Van Damme, Pierre \& Leuridan, Elke(2017) Effect of Prepregnancy Pertussis Vaccination in Young Infants. In: JOURNAL OF INFECTIOUS DISEASES, 215(12), p. 1855-1861

DOI: 10.1093/infdis/jix176

Handle: http://hdl.handle.net/1942/24324 


\section{Effect of Pre-pregnancy Pertussis Vaccination in Young Infants}

2 Kirsten Maertens $(\mathrm{Msc})^{1}$, Thao Mai Phuong Tran $(\mathrm{Msc})^{2}$, Niel Hens (Prof) $)^{2,3}$, Pierre Van Damme (Prof) ${ }^{1}$,

$3 \quad$ Elke Leuridan (Prof) ${ }^{1}$

$4{ }^{1}$ Center for the Evaluation of Vaccination, Vaccine \& Infectious Diseases Institute, University of Antwerp,

5 Universiteitsplein 1, 2610 Wilrijk, Belgium

$6{ }^{2}$ Interuniversity Institute for Biostatistics and statistical Bioinformatics (I-Biostat), Hasselt University,

7 Belgium, Martelarenlaan 42, 3500 Hasselt, Belgium

$8{ }^{3}$ Centre for Health Economic Research and Modelling Infectious Diseases, Vaccine \& Infectious Disease

9 Institute, University of Antwerp, Universiteitsplein 1, 2610 Wilrijk, Belgium

10 Running title: Pre-pregnancy Pertussis vaccination

11 Word count: 3340 words

12 Word count abstract: 198 words

14 Short summary: Post-partum Tdap vaccination increases significantly maternal antibody concentrations

15 at consecutive delivery, and blunting of the later born siblings' immune responses occurs, similar to the

16 effect of Tdap vaccination during pregnancy. 


\section{Abstract}

19 Background

20 Maternal pertussis antibodies can hamper infant immune responses to pertussis vaccines. The effect of

21 offering a maternal tetanus, diphtheria, acellular pertussis (Tdap) booster between 2 consecutive

22 pregnancies is investigated.

23 Methods

24 A prospective study was conducted (Belgium, 2008-2014) on kinetics of maternal pertussis antibodies in

25 unvaccinated women and their infants (group $A, N=86$ ) and in siblings (group $B, N=58$ ), born after the

26 woman received a Tdap vaccination. Anti-Pertussis Toxin (PT), anti-Filamentous Haemaglutinin (FHA) and

27 anti-Pertactin (Prn) antibodies were measured in maternal blood before and after vaccination and at

28 both deliveries, in cord blood from both siblings and before and after a priming series of infant pertussis

29 vaccines.

30 Results

31 All pertussis antibodies were significantly higher in group B siblings at birth, even with growing time

32 interval since maternal vaccination. Blunting of the infant pertussis vaccine response was detected in

33 group B siblings.

34 We estimated a maximum time interval between repeat Tdap doses in adult women in order to have a

35 beneficial effect for the consecutive infant.

36 Conclusions

37 Pre-pregnancy Tdap vaccination increases significantly maternal antibody concentrations at consecutive

38 delivery. However, similarly to the effect of Tdap vaccination during pregnancy, blunting of the infant

39 immune responses occurs after a pre-pregnancy immunisation. 
40 Key words: maternal antibodies; pertussis; vaccination; pre-pregnancy; blunting 


\section{$42 \quad$ Background}

43 Despite universal infant vaccination programs against Bordetella pertussis, there is an increase in

44 reported whooping cough cases, particularly in industrialized countries. Most outbreaks occur in

45 adolescents and young adults [1] and they are representing a source of infection for unvaccinated infants

46 and newborns. Highest incidence of pertussis disease is recorded in very young children who havenot

47 yet been (fully) vaccinated and/ or did not receive sufficient maternal antibodies [2].

48 At first, cocoon vaccination was recommended to protect young infants from disease, but cost

49 effectiveness studies, as well as difficulties to reach high coverages among all persons in contact with

50 young infants, discouraged the strategy [3]. Since 2012, maternal vaccination during pregnancy is a

51 recommended strategy in an increasing number of (industrialized) countries to protect young infants

52 from disease. Many countries advise to immunize at every pregnancy, since antibodies are rapidly

53 waning after an adult booster dose [4], and a high concentration of maternal antibodies is needed for

54 transfer of antibodies from mother to fetus during pregnancy [5]. For pertussis, a correlate of protection

55 is not well defined, yet high concentrations of anti-Pertactin (Prn) and mainly anti-Pertussis Toxin (PT)

56 antibodies, are related to protection [6]. After birth, maternal antibodies decrease rapidly in the

57 offspring, mostly within two months [7].

58 The present paper reports results of a prospective study on the transfer and persistence of maternal

59 antibodies in newborns, whenever a tetanus, diphtheria and pertussis (Tdap) booster vaccination is given

60 to women between two consecutive pregnancies. Previously, we reported an interim analysis on a

61 subsample of this cohort study [8]. The primary aim of the study is to evaluate whether a pre-pregnancy

62 booster helps to increase antibody concentrations to pertussis in neonates, thereby closing the

63 susceptibility gap. A secondary aim is to evaluate possible blunting of the infant immune responses to

64 pertussis containing vaccines after a pre-pregnancy maternal booster vaccination, similar to blunting of

65 the infant immune responses after Tdap administration in pregnancy [9]. In addition, modelling theanti- 
66 PT IgG antibody concentrations enables drafting possible recommendations regarding the timing of

67 consecutive maternal booster doses.

\section{Methods}

\section{Study design}

70 A prospective multicenter study was conducted in Antwerp, Belgium, between 2008 and 2014, in

71 accordance with the Helsinki Declaration, ICH-GCP and procedures established by Belgian law. Ethical

72 approval was obtained (University Hospital of Antwerp). Women participating in a study on maternal

73 antibody kinetics were recruited [10-12]. Informed consent was obtained from the women and from

74 both parents of the participating children. For the women, the exclusion criteria were pertussis

75 vaccination within ten years prior to study participation, immunological disorders and recent

76 administration of immunoglobulins prior to vaccination. Exclusion criteria for the children were low birth

77 weight $(<2400 \mathrm{~g})$, prematurity ( $<36$ weeks gestation) and immunological disorders. Serum sampleswere

78 taken from all women at delivery $(10 \mathrm{~mL})$, from the cord $(10 \mathrm{~mL})$, and from the infant at 1 month of age,

796 or 9 months of age (randomly) and 12 months of age ( $2 \mathrm{cc}$ ) (Group A). Time points were chosen based

80 on waning of maternal measles antibodies [10].

81 Women were then offered a tetanus, diphtheria, acellular pertussis (Tdap) vaccine (Boostrix ${ }^{\circledR}$, GSK

82 Biologicals, Rixensart, Belgium) after the first delivery. One month after vaccination, a blood sample (10

$83 \mathrm{~mL}$ ) was taken. At consecutive delivery, blood was again taken from the woman and the cord and later

84 on from the sibling at 1 month of age, 6 or 9 months (randomly and equal to the older sibling) and 12

85 months of age (Group B).

86 All infants were vaccinated within the standard Belgian vaccination schedule with the hexavalent aP

87 containing vaccine Infanrix $\mathrm{Hexa}^{\circledR}$ (GSK Biologicals, Rixensart, Belgium) at 8, 12 and 16 weeks. 
88 An extended questionnaire collected information on obstetrical risk factors, demographics, vaccination

89 history, and general medical history. Growth parameters, breastfeeding data, immunization data, and

90 medical history were collected at each visit.

\section{Study vaccines}

92 Licensed Tdap vaccine (Boostrix ${ }^{\circledR}$, GSK Biologicals, Rixensart, Belgium) was used to immunize women in

93 the deltoid muscle. Boostrix ${ }^{\circledR}$ contains 5 Lf of tetanus toxoid (TT), 2.5 Lf of diphtheria toxoid (DT), $8 \mu$ g of

94 inactivated PT, $8 \mu \mathrm{g}$ of FHA and $2.5 \mu \mathrm{g}$ of Prn. Infants were vaccinated with the hexavalent vaccine

95 Infanrix Hexa ${ }^{\circledR}$ (GSK Biologicals, Rixensart, Belgium), containing 25 Lf of DT, 10 Lf of TT, $25 \mu \mathrm{g}$ PT, $25 \mu \mathrm{g}$

96 FHA and 8 mg Prn, inactivated poliovirus, hepatitis B surface antigens and Haemophilus influenzae type B

97 polysaccharide.

$98 \quad$ Laboratory

99 All serum samples were centrifuged at $2000 \mathrm{rpm}$ within 24 hours after withdrawal, and stored

100 between $-20^{\circ} \mathrm{C}$ and $-40^{\circ} \mathrm{C}$. Serum leftovers from women and infants in group A were selected foranalysis

101 in view of the inclusion of their siblings (group B siblings). An in-house ELISA was used to test all samples

102 for anti-PT, anti-FHA and anti-Prn IgG antibodies at GSK Biologicals, Belgium. The limit of detection of the

103 assay was $5 \mathrm{EU} / \mathrm{mL}$ for all three antibodies. For women, a booster response was defined as a post-

104 vaccination antibody concentration $\geq 20 \mathrm{EU} / \mathrm{mL}$ with a pre-vaccination antibody concentration $<5$

$105 \mathrm{EU} / \mathrm{mL}$, a post-vaccination rise of at least 4 times the pre-vaccination antibody concentration in subjects

106 with a pre-vaccination antibody concentration $\geq 5 \mathrm{EU} / \mathrm{mL}$ and $<20 \mathrm{EU} / \mathrm{mL}$; or at least twice the pre-

107 vaccination antibody concentration in subjects with a pre-vaccination antibody concentration $\geq 20$

$108 \mathrm{EU} / \mathrm{mL}$. The pre-vaccination value was the maternal sample taken at the first delivery. 
111 Antibody geometric mean concentrations (GMCs) with $95 \% \mathrm{Cl}$ were calculated. Statistical tests included

112 parametric tests: (paired) t-tests and chi-square tests and their non-parametric alternatives: (paired)

113 Wilcoxon tests and Fisher exact tests. The analysis was performed using SPSS statistical software version 11423.0.

115 Non-linear mixed effect models (NLMM) were employed to model the dynamics of anti-PT antibodies in 116 both mothers and infants. Anti-PT antibodies were chosen because they, as well as anti-Prnantibodies, 117 correlate with protection [13]. The model building procedure is motivated in the Appendix. The models 118 were fitted using Monolix software [14]. The anti-PT antibody values less than $5 \mathrm{EU} / \mathrm{mL}$ are treated as 119 left-censored data. Results of the NLMM are expressed as medians and interquartile range (IQR), 120 because of possible asymmetry for the quantities under study as well as for ease of interpretation. Figure

1211 in the Appendix shows a presumed visualization of the dynamics of the anti-PT antibody levels in the 122 infants, indicating the different slopes and rates, used in the NLMM model.

123 Finally, a robust simple linear regression model was fitted to investigate the association between the 124 antibody levels in women at delivery and in the cord.

125 All the rates mentioned in the manuscript are exponential decay or growth rates expressed in months, 126 unless indicated differently. A significance level of $5 \%$ was used for all analyses. Blunting of vaccine 127 immune responses was defined as a significantly lower GMC of pertussis specific IgG antibodies ingroup 128 B siblings. 


\section{Results}

\section{General characteristics of the study population}

133 In total, 86 women received a Tdap vaccine after a first pregnancy. All women had been vaccinated

134 against pertussis during childhood with whole cell pertussis (WP) containing vaccines and received no

135 documented pertussis booster for at least 10 years prior to the study booster. Of the 86 vaccinated

136 women, 58 women became pregnant again and delivered within the study period.

137 The median interval between the first delivery and the Tdap vaccination was 16.07 months (min-max:

138 8.46-43.31). The median interval between the Tdap booster and the consecutive delivery was 16.8

139 months (min-max: 6.20-56.49). Three women had a negative pregnancy test at the time of vaccination

140 but were pregnant 1 month later, despite contraceptive advice. The mean age of the women was 29.97

141 years at first, and 32.04 years at consecutive delivery. Mean duration of both pregnancies was

142 comparable. No significant difference in birth weight and length of both siblings was seen, nor gender

143 ratio differences (Table 1).

144 Insert Table 1 here.

145 Laboratory results

$146 \quad$ a) Maternal results

147

148 Table 2 summarizes the GMC of IgG antibody concentrations to PT, FHA and Prn in all women at delivery

149 of the first born infants (Group A) (= pre-vaccination sample of the mother), 1 month post-booster

150 vaccination, and at the moment of delivery of the Group B siblings.

151 Insert Table 2 here.

152 At baseline, 93\% of the women had detectable anti-FHA IgG antibodies and 79\% had detectable anti-Prn 153 IgG antibodies, while 52\% had anti-PT IgG antibody concentrations > $5 \mathrm{EU} / \mathrm{mL}$. $16 \%$ of participating 
women were seronegative for both anti-PT and anti-Prn antibodies at baseline. One month after

155 vaccination, $97.7 \%$ of the women showed a booster response to FHA and Prn while $90.7 \%$ showed a

156 booster response to PT. All but one woman responded with a rise in all pertussis-specific antibodies, and

157 there was no difference in magnitude of the response for anti-PT responses, nor for anti-Prn responses,

158 between seronegative women and seropositive women pre-vaccination. At the next delivery, the mean

159 maternal antibody levels for all 3 antigens had declined significantly $(p<0.001)$ compared to one month

160 after vaccination, but were still significantly higher compared to baseline concentrations forall

161 antibodies $(p<0.001)$. In 8 women, the anti-PT antibody concentration dropped below the threshold of 5

$162 \mathrm{EU} / \mathrm{mL}$ at the consecutive delivery.

b) Cord results

Table 3 shows the transplacental transport ratio in both cohorts of infants. In general, GMC in cord blood exceeds GMC in the mother at delivery for both siblings. Ratios did not significantly differ at consecutive pregnancies.

Insert Table 3 here.

172 Group B siblings have significantly higher antibody levels to all pertussis specific antigens at birth 173 compared to group A infants, lasting up to the age of 1 month, before the start of the infant vaccination 174 program. After three doses of primary vaccination, all antibody concentrations are consistently lower in 175 group B siblings (month 6). For anti-FHA and anti-Prn, the IgG concentrations are significantly lower at 176 the ages of 9 and 12 months. For anti-PT on the other hand, the antibody concentrations are only 177 significantly lower at the age of 12 months (Table 2). 
a) Maternal results

181 Based on the NLMM, the median time for anti-PT antibodies in women to decrease by $50 \%$ (i.e. the half-

182 life) is 15.87 months (IQR: $14.86-16.74)$. The age of the mother at vaccination had no significanteffect 183 on this half-life.

184 Since there is no correlate of protection for pertussis, it is unclear how high the maternal antibody 185 concentration at delivery should be in order to protect the offspring. Figure 2 shows the median time 186 point (with IQR) whenever antibody concentrations in women fall below a pre-specified level (from 5 - 25

$187 \mathrm{EU} / \mathrm{mL}$ ) after a booster vaccination. After a median time of 55.56 months (IQR: $51.76-59.33$ ) post-

188 vaccination, the anti-PT IgG antibody levels in women decline below the threshold value of $5 \mathrm{EU} / \mathrm{mL}$, and 189 after a median time of 30.24 months (IQR: $27.77-33.25$ ), the anti-PT IgG antibody levels have reached 190 the threshold of $15 \mathrm{EU} / \mathrm{mL}$. For comparison, the GMC of maternal antibodies at delivery of the group B 191 siblings was $13,5 \mathrm{IU} / \mathrm{mL}$, and this titer corresponded with possibly enough maternal antibodies in the 192 cord to protect young infants.

Insert Figure 1 here

b) Infant results

195 The results of the NLMM confirm that siblings in group B have a significantly higher antibody 196 concentration at birth (i.e. $A_{0}$ in Figure 1)) compared to group A infants. After birth and before the start 197 of the infant vaccination program, maternal antibody levels decrease very fast in both groups (Figure 3): 198 the median time for anti-PT antibody levels in group A infants to fall below $5 \mathrm{EU} / \mathrm{mL}$, is 1.21 months (IQR: $1991.08-1.36$ ), while it takes about 2.21 months (IQR: $2.09-2.39$ ) to drop below $5 \mathrm{EU} / \mathrm{mL}$ in group B 200 siblings. The half-life of maternal anti-PT antibody levels in infants is approximately 5 weeks (33 days and 20130 days for children group A and B, respectively). 
Infants in group B have a significantly lower increase rate (denoted by $\gamma$ in Figure 1 ) of anti-PT IgG antibody levels after priming with 3 vaccine doses. The time point $h$, at which we observe the highest antibody concentration before waning of antibodies, is estimated to be at 6.82 months (IQR: 6.637.01). The last scheduled vaccination was administered at 16 weeks of age, but the effect of 3 subsequent primary doses made the antibody levels still increase approximately 2.82 months after the 207 last dose.

We included gender and birthweight of the infants, centered around its mean, in the NLMM: boys were born with significantly higher anti-PT antibody concentrations compared to girls in both groups. Time point $h$ in boys is significantly lower (5.55 months (IQR: $5.33-5.77)$ ), compared to the estimated value of 7.06 months [6.84 - 7.28] for girls. Newborns with higher birth weight tend to have higher estimated decay rates $\beta$ albeit this small difference is not clinically relevant.

c) Correlation between maternal and infant antibody levels

215 The robust simple linear regression model shows a significant positive association between the anti-PT 216 IgG antibody levels in women at delivery and in the cord. The predicted anti-PT IgG antibody levels in the 217 cord of the infant whose mother had anti-PT IgG antibody levels at delivery of 5, 10, 15, 20 and 25 $218 \mathrm{EU} / \mathrm{mL}$, are respectively: $10.43 \mathrm{IU} / \mathrm{mL}$ [9.31 - 11.55], 18.80 [17.04-20.56], 26.53 [23.87- 29.19], 33.88 $219[30.12-37.64]$ and $40.96[35.97-45.95] \mathrm{EU} / \mathrm{mL}$.

220 In order to evaluate the possible interval between several booster doses of Tdap in 1 woman, the 221 correlation of maternal antibodies at delivery, with titers in cord and the loss of these maternal antibodies 222 in the young infant over time (as a measure for protection from disease) could offer insights in the need 223 for repeat booster, also considering possible interference of infant immune responses on vaccines. If, at 224 delivery, the anti-PT antibody titer in the mother is $15 \mathrm{EU} / \mathrm{mL}$, it takes roughly 2.5 months in infants for 225 anti-PT antibody levels to decline below $5 \mathrm{EU} / \mathrm{mL}$ and 1 month to fall below $10 \mathrm{EU} / \mathrm{mL}$ (Figure2). 
226 Insert Figure 2 here

227 
229 At baseline, $48 \%$ of the women in the present long term follow up study, had undetectable anti-PT 230 antibody levels, indicating the lack of maternal antibodies to be transferred and hence the lack of 231 protection offered at birth. After a post-partum Tdap booster vaccination, good humoral responses 232 were measured. Yet, similarly to other vaccination studies with Tdap in adults [15, 16], [17], a rapid drop

233 in antibody concentrations is measured after vaccination: the half-life of anti-pertussis antibodies is 234 estimated to be $\mathbf{1 5 . 9 0}$ months. However, median antibody concentrations remained significantly higher 235 in women at a next delivery, even if the booster dose was offered more than 2 years before consecutive 236 delivery. And significantly increased anti-pertussis antigen specific maternal antibodyconcentrations 237 were still encountered in that offspring, born after the booster dose. In 13\% (8/58) of the women, the 238 anti-PT IgG had declined below the lower limit of $5 \mathrm{IU} / \mathrm{mL}$ by the time of the next delivery, indicating 239 possible susceptibility to disease for the young infant, and underlining the importance of repetitive 240 boosting with each new pregnancy.

241 Transplacental transport in children born before and after the pre-pregnancy booster vaccination was 242 equally effective. Previously, adequate transport has been described in prospective cohorts $[9,18,19]$.

243 Yet, this is the first study describing transplacental transport in siblings, and no effect of vaccinationor 244 parity was found on the adequacy of the transport. We would like to stress that our cohort consisted of 245 healthy pregnant women with mostly healthy pregnancies, while transplacental transport can be 246 influenced by placental dysfunction or disease. We confirm in the present study the recently published 247 Swiss data that the presence of high titers of maternal antibodies during the entire pregnancy, or a long 248 period thereof, results in adequate transport and high concentrations at birth [20].

249 Using a NLMM, we were able to calculate the impact of time lapse between repeat boosters in healthy 250 adult women on transferred antibody levels towards the fetus. Based on these results, the time frame to 
251 be considered between consecutive pertussis booster vaccines should be no longer than 30 months

252 (roughly 2.5 years), in order to have median anti-pertussis antibody concentrations in pregnantwomen

253 as high as $15 \mathrm{EU} / \mathrm{mL}$ at the moment of delivery. This level corresponds to anti-PT IgG antibody levels in

254 cord blood of $26.53 \mathrm{EU} / \mathrm{mL}$ in the present study (IQR: $23.87-29.19$ ). These results are based on the

255 assumption that the estimated decay rate derived from our model is the same as the one we would

256 obtain with a larger number of samples (See Appendix), and on the assumption that the positive effect

257 that we found in group B siblings, is protective against disease. This is not a recommendation, yeta

258 finding that has to be confirmed based on additional persistence studies, and possibly including

259 information on antibody levels at multiple time points to inform antibody dynamics. In addition, similar

260 calculations when vaccination is offered during pregnancy are needed and planned.

261 Infants born after a pre-pregnancy booster had significantly higher anti-PT GMC at birth that endured up

262 to one month of age, thus closing potentially the susceptibility gap for infection. These results are in line

263 with on the effect of Tdap vaccination during pregnancy $[9,18,19]$. The median half-life of anti-PTIgG

264 antibodies in infants is relatively short (5 weeks) which is again in line with literature [21-23]. Since no

265 correlate of protection is known for pertussis [13], it is not sure whether the elevated antibody

266 concentrations in the second born cohort are sufficiently protective against disease, or whether the

267 blunting later in infant life, after a primary series of three vaccines, is meaningful in terms of protection.

268 Indeed, the effect of these maternal antibodies on the immune responses to the primaryinfant

269 vaccination, is still detectable in group B siblings born after a pre-pregnancy booster dose. This so-called

270 blunting effect has been described after maternal Tdap vaccination during pregnancy $[9,18,19,24]$,

271 however this is the first study to describe the same effect after a pre-pregnancy (or post partum) booster

272 dose. What we measure is in fact the effect of cocoon vaccination on offspring that is born later on.

273 Effectiveness data are lacking whether the elevated titer of maternal antibodies is protecting young

274 infants from disease. 
275 At months 6 and 9, after primary vaccination, differences between both siblings are not always

276 significant for all 3 tested antibody types, perhaps due to the small sample size. GMC in infants at 12

277 months of age, measured in previous studies [25], are comparable with GMC in children at 1 month of

278 age after a pre-pregnancy booster, suggesting that GMC in group B siblings at birth would be compatible

279 with clinical protection.

280 The present study shows that also countries with a recommendation for cocoon vaccination, or with

281 repeated booster recommendations, should be aware that significantly higher antibodyconcentrations

282 are transferred to the offspring, thereby leading to possible early life protection but also potential

283 interference of the maternal antibodies with the infant's immune response. Data could be used for cost-

284 benefit calculations for subsequent doses in women of childbearing age.

286 There are a few shortcomings in this study. There is a rather low number of infants born after the

287 booster dose as a result of the difficulty to plan this upfront. Besides, the study was not powered to

288 detect small differences in antibody levels between both siblings, with growing time interval between

289 vaccination and delivery of a second born child. In addition, the time points of blood samplingwere

290 chosen according to the original study on measles antibody kinetics, and those were likely not the most

291 optimal time points for pertussis responses. Nevertheless, pre- and post-vaccination sampleswere

292 available and are always taken at the same time points. In order to understand better the relevance of

293 the findings, measurement of the functionality of the antibodies, would be valuable, ideallycomparing

294 functionality of antibodies elicited during pregnancy, and as in this project, in a non-pregnant status.

295 To conclude, the present study is the first study describing the effects of a pre-pregnancy Tdap booster

296 vaccine, comparing kinetics of pertussis antibodies between siblings born before and after the booster 
297 dose. The maternal antibodies at birth are significantly higher in the second born siblings, even with a 298 growing time interval between booster and subsequent delivery.

299

300 


\section{Funding}

303 This work was supported by an unrestricted grant from GSK Biologicals to perform this study. ELis

304 beneficiary holder of a postdoctoral mandate fellowship from the FWO (FWO 12D6114N). NHgratefully

305 acknowledges support from the University of Antwerp scientific chair in Evidence-Based Vaccinology,

306 financed in 2009-2016 by an unrestricted gift from Pfizer and in 2016 from GSK.

\section{Acknowledgements}

308 Authors express their gratitude to Mrs Aline Bontenakel, senior study nurse, for performing all blood 309 samplings. The obstetricians and pediatricians of the Antwerp University Hospital (UZA), the hospitals of 310 the Gasthuiszusters of Antwerp and the ZNA hospitals of Antwerp are gratefully acknowledged for their

311 participation. EL received an unrestricted grant from GSK Biologicals to perform this study and is

312 beneficiary holder of a postdoctoral mandate fellowship from the FWO (FWO 12D6114N). NH gratefully

313 acknowledges support from the University of Antwerp scientific chair in Evidence-Based Vaccinology,

314 financed in 2009-2016 by an unrestricted gift from Pfizer and in 2016 from GSK.

\section{Conflict of interest}

316 Potential conflicts of interest: EL was member of an advisory board on Pertussis vaccines (GSK

317 Biologicals, 2016, non-financial support). The University of Antwerp obtains grants from severalvaccine

318 manufacturers for the conduct of vaccine trials for which PVD is the investigator. NH is holder of the

319 scientific chair of evidence-based vaccinology sponsored by an unrestricted gift from Pfizer and GSK.

\section{$320 \quad$ Meetings}

321 Preliminary data have been presented at the INMIS congress in November 2015 in The Gambia. Anda

322 first interim analysis has been published in: 'Effect of a prepregnancy pertussis booster dose on maternal 
323 antibody titers in young infants. Leuridan E, Hens N, Peeters N, de Witte L, Van der Meeren O, Van

324 Damme P. Pediatr Infect Dis J. 2011 Jul;30(7):608-10.'

325 Corresponding author

326 Prof. Dr. Elke Leuridan

327 University of Antwerp, Centre for the Evaluation of Vaccination, Vaccine \& Infectious Disease Institute

328 Universiteitsplein 1, 2610 Wilrijk, Belgium, Tel: +0032 32652885, elke.leuridan@uantwerp.be 
333 1. Wendelboe AM, Van Rie A, Salmaso S, Englund JA. Duration of immunity against pertussis afternatural

334 infection or vaccination. The Pediatric infectious disease journal 2005;24:S58-61.

335 2. Roehr B. Whooping cough outbreak hits several US states. British Medical Journal 2010;341:c4627.

336 3. Blain AE, Lewis $M$, Banerjee $E$, et al. An Assessment of the Cocooning Strategy for Preventing Infant

337 Pertussis-United States, 2011. Clinical infectious diseases : an official publication of the Infectious

338 Diseases Society of America 2016; 63:S221-S6.

339 4. Halperin BA, Morris A, Mackinnon-Cameron D, et al. Kinetics of the antibody response to tetanus-

340 diphtheria-acellular pertussis vaccine in women of childbearing age and postpartum women. Clin Infect

341 Dis 2011; 53:885-92.

342 5. Healy CM, Munoz FM, Rench MA, Halasa NB, Edwards KM, Baker CJ. Prevalence of pertussis antibodies

343 in maternal delivery, cord, and infant serum. Journal of Infectious Diseases 2004;190:335-40.

344 6. Taranger J, Trollfors B, Lagergard T, et al. Correlation between pertussis toxin IgG antibodiesin

345 postvaccination sera and subsequent protection against pertussis. The Journal of infectious diseases

$346 \quad 2000 ; 181: 1010-3$.

347 7. Healy CM, Rench MA, Baker CJ. Importance of timing of maternal combined tetanus, diphtheria, and

348 acellular pertussis (Tdap) immunization and protection of young infants. Clin Infect Dis 2013;56:539-44.

349 8. Leuridan E, Hens N, Peeters N, de Witte L, Van der Meeren O, Van Damme P. Effect of aPrepregnancy

350 Pertussis Booster Dose on Maternal Antibody Titers in Young Infants. The Pediatric infectious disease

351 journal 2011; 30:608-10.

352 9. Maertens K, Cabore RN, Huygen K, Hens N, Van Damme P, Leuridan E. Pertussis vaccination during

353 pregnancy in Belgium: Results of a prospective controlled cohort study. Vaccine 2016;34:142-50.

354 10. Leuridan E, Hens N, Hutse V, leven M, Aerts M, Van Damme P. Early waning of maternalmeasles

355 antibodies in era of measles elimination: longitudinal study. British Medical Journal 2010;340:c1626. 
357 children up to the age of 1 year. European journal of pediatrics 2012; 171:1167-73.

358 12. Leuridan E, Hens N, Hutse V, Aerts M, Van Damme P. Kinetics of maternal antibodies against rubella 359 and varicella in infants. Vaccine 2011;29:2222-6.

360 13. Plotkin SA. Correlates of protection induced by vaccination. Clin Vaccine Immunol 2010; 17:1055-65.

361 14. Lixoft. Monolix methodology: version 4.3.2. A software for the analysis of nonlinear mixed effect 362 models. . Available at: http://download.lixoft.com/data/packages/mlx-

363 4.3.2/guides/monolixMethodology.pdf. Accessed 02/12/2016.

364 15. Weston W, Messier M, Friedland LR, Wu X, Howe B. Persistence of antibodies 3 years after booster 365 vaccination of adults with combined acellular pertussis, diphtheria and tetanus toxoids vaccine. Vaccine $366 \quad 2011 ; 29: 8483-6$.

367 16. Zimmermann U, Gavazzi G, Richard P, Eymin C, Soubeyrand B, Baudin M. Immunogenicity and safety 368 of a booster dose of diphtheria, tetanus, acellular pertussis and inactivated poliomyelitis vaccine (Tdap369 IPV; Repevax) administered concomitantly versus non-concomitantly with an influenza vaccine(Vaxigrip) 370 to adults aged >/=60 years: an open-label, randomised trial. Vaccine 2013;31:1496-502.

371 17. Dalby T, Petersen JW, Harboe ZB, Krogfelt KA. Antibody responses to pertussis toxin display different 372 kinetics after clinical Bordetella pertussis infection than after vaccination with an acellular pertussis 373 vaccine. J Med Microbiol 2010; 59:1029-36.

374 18. Munoz FM, Bond NH, Maccato M, et al. Safety and immunogenicity of tetanus diphtheria and 375 acellular pertussis (Tdap) immunization during pregnancy in mothers and infants: a randomizedclinical 376 trial. JAMA : the journal of the American Medical Association 2014;311:1760-9.

377 19. Hoang HT, Leuridan E, Maertens K, et al. Pertussis vaccination during pregnancy in Vietnam: Results 378 of a randomized controlled trial Vaccine $2016 ; 34: 151-9$. 
379 20. Eberhardt CS, Blanchard-Rohner G, Lemaitre B, et al. Maternal immunization earlier in pregnancy

380 maximizes antibody transfer and expected infant seropositivity against pertussis. Clinical infectious

381 diseases : an official publication of the Infectious Diseases Society of America 2016.

382 21. Vilajeliu A, Ferrer L, Munros J, et al. Pertussis vaccination during pregnancy: Antibody persistencein

383 infants. Vaccine 2016; 34:3719-22.

384 22. Van Savage J, Decker MD, Edwards KM, Sell SH, Karzon DT. Natural history of pertussis antibody in

385 the infant and effect on vaccine response. The Journal of infectious diseases 1990;161:487-92.

386 23. Van Rie A, Wendelboe AM, Englund JA. Role of maternal pertussis antibodies in infants. The Pediatric

387 infectious disease journal 2005; 24:S62-5.

388 24. Ladhani SN, Andrews NJ, Southern J, et al. Antibody responses after primary immunization ininfants

389 born to women receiving a pertussis-containing vaccine during pregnancy: single arm observational

390 study with a historical comparator. Clinical infectious diseases : an official publication of the Infectious

391 Diseases Society of America 2015; 61:1637-44.

392 25. Tichmann I, Grunert D, Habash S, et al. Persistence of antibodies in children primed with two

393 different hexavalent diphtheria, tetanus, acellular pertussis, hepatitis B, inactivated poliovirusand

394 Haemophilus influenzae type B vaccines and evaluation of booster vaccination. Human vaccines 2006;

$395 \quad 2: 249-54$ 
403

404

405

406 
409 Table 1: Demographic characteristics of the participating women and infants

410 Table 2: Geometric Mean Concentration (GMC) with 95\% confidence interval ( $95 \% \mathrm{Cl}$ ) for antibody

411 concentrations against Filamentous Hemagglutinin (FHA), Pertactin (Prn) and Pertussis Toxin (PT) in

412 women and infants at different time points, expressed in Elisa Units per milliliter (EU/mL).

413 There was not enough sample available anymore from all women at the delivery of the first bornchild.

414 Statistical test used: unpaired t-test.

415 Figure 1: The median time point (with their IQR) whenever the anti-PT IgG antibodies in women, after 416 booster vaccination, decline below a pre-specified threshold ( $x$-axis) between 5 and $25 \mathrm{EU} / \mathrm{mL}$.

417 Figure 2: Decline of the maternal antibodies during the first months of life, in both groups of infants 418 (group A infants expressed in red and group B siblings expressed in blue). The solid lines show the 419 median time points (with their IQR in dotted lines, y-axis) whenever the maternal anti-PT IgG antibodies

420 in infants start to decline below a pre-specified threshold ( $x$-axis) between 5 and $10 \mathrm{EU} / \mathrm{mL}$ (when no 421 infant vaccination is performed).

422 Table 3: Transplacental transport ratio for Pertussis Toxin, Filamentous haemagglutinin and pertactin 423 antibodies in group A and group B infants. Statistical test used: unpaired t-test. 


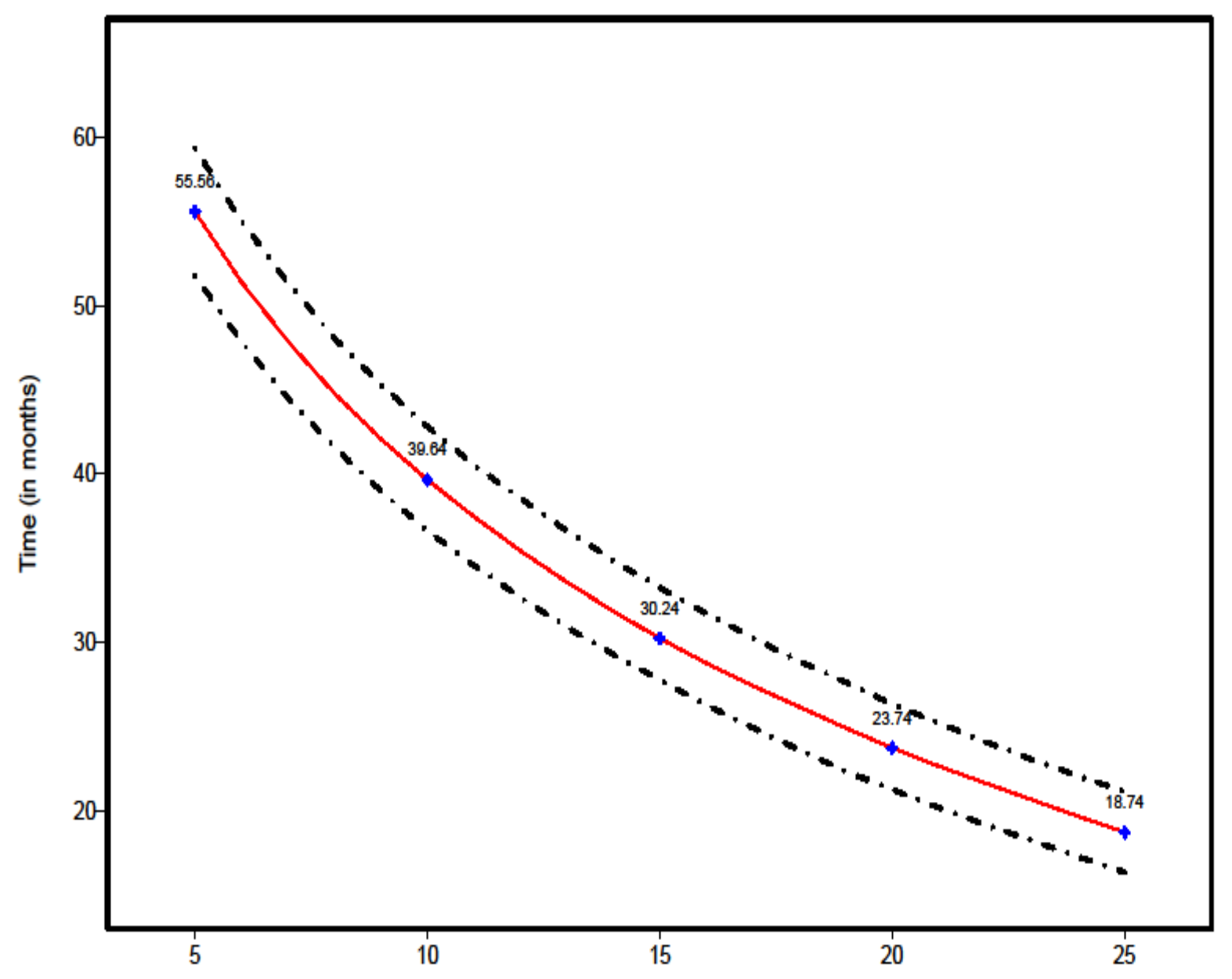

Anti-PT IgG antibodies in pregnant women

Figure 1: The median time point (with their IQR) whenever the anti-PT IgG antibodies in women, after booster vaccination, decline below a pre-specified threshold (x-axis) between 5 and $25 \mathrm{EU} / \mathrm{mL}$. 


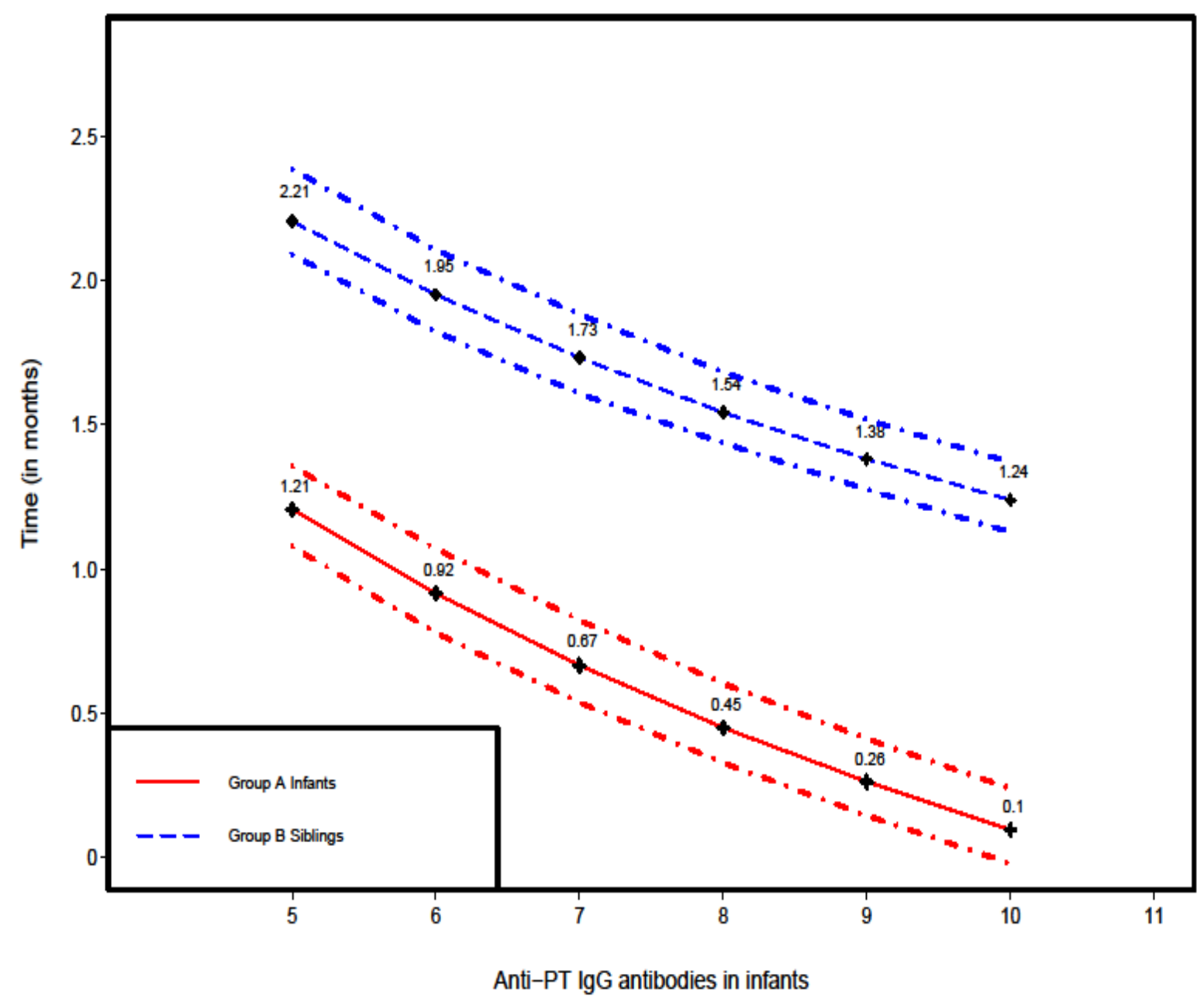

Figure 2: Decline of the maternal antibodies during the first months of life, in both groups of infants (group A infants expressed in red and group B siblings expressed in blue). The solid lines show the median time points (with their IQR in dotted lines, y-axis) whenever the maternal anti-PT IgG antibodies in infants start to decline below a pre-specified threshold (x-axis) between 5 and $10 \mathrm{EU} / \mathrm{mL}$ (when no infant vaccination is performed). 


\begin{tabular}{|c|c|c|c|}
\hline \multicolumn{4}{|l|}{ Demographic characteristics women } \\
\hline Mean age at vaccination in years (SEM) & \multicolumn{3}{|c|}{$30.53(0.36)$} \\
\hline $\begin{array}{l}\text { Median interval between delivery first child and } \\
\text { vaccination in months (IQR) }\end{array}$ & \multicolumn{3}{|c|}{$16.07(11.90)$} \\
\hline $\begin{array}{l}\text { Mean interval between vaccination - blood } \\
\text { sampling in month (SEM) }\end{array}$ & \multicolumn{3}{|c|}{$1.00(0.01)$} \\
\hline \multirow{2}{*}{$\begin{array}{l}\text { Median interval between vaccination and } \\
\text { delivery child } 2 \text { in months (IQR) }\end{array}$} & \multicolumn{3}{|c|}{$16.80(15.48)$} \\
\hline & Delivery infant A & Delivery sibling B & p-value \\
\hline $\begin{array}{l}\text { Mean age of the mother at delivery in years } \\
\text { (SEM) }\end{array}$ & $29.97(0.37)$ & $32.04(0.41)$ & Not of interest \\
\hline Gestational age at birth in weeks (SEM) & $39.43(0.13)$ & $39.25(0.17)$ & 0.395 \\
\hline $\begin{array}{l}\text { Mean duration of breastfeeding in months } \\
\text { (SEM) }\end{array}$ & $4.43(0.31)$ & $4.72(0.40)$ & 0.555 \\
\hline Demographic characteristics infants & Group A infants & Group B siblings & p-value \\
\hline Gender, No. Male (\%)/ Female (\%) & $39(45.88) / 46(54.12)$ & $30(51.72) / 28(48.28)$ & 0.383 \\
\hline $\begin{array}{l}\text { Mean age at blood sample month } 1 \text { in months } \\
\text { (SEM) }\end{array}$ & $1.01(0.01)$ & $1.02(0.01)$ & 0.758 \\
\hline $\begin{array}{l}\text { Mean age at blood sample month } 6 \text { in months } \\
\text { (SEM) }\end{array}$ & $6.01(0.03)$ & $6.56(0.11)$ & 0.256 \\
\hline $\begin{array}{l}\text { Mean age at blood sample month } 9 \text { in months } \\
\text { (SEM) }\end{array}$ & $9.00(0.02)$ & $9.19(0.11)$ & 0.120 \\
\hline $\begin{array}{l}\text { Mean age at blood sample month } 12 \text { in months } \\
\text { (SEM) }\end{array}$ & $12.04(0.02)$ & $12.46(0.10)$ & $<0.001$ \\
\hline $\begin{array}{l}\text { Mean age at hexavalent vaccine dose } 1 \text { in } \\
\text { months (SEM) }\end{array}$ & $2.25(0.04)$ & $2.13(0.10)$ & 0.532 \\
\hline $\begin{array}{l}\text { Mean age at hexavalent vaccine dose } 2 \text { in } \\
\text { months (SEM) }\end{array}$ & $3.43(0.05)$ & $3.49(0.10)$ & 0.572 \\
\hline $\begin{array}{l}\text { Mean age at hexavalent vaccine dose } 3 \text { in } \\
\text { months (SEM) }\end{array}$ & $4.62(0.07)$ & $4.73(0.12)$ & 0.450 \\
\hline $\begin{array}{l}\text { Mean interval between hexavalent vaccine dose } \\
3 \text { and blood sample month } 6 \text { in months (SEM) }\end{array}$ & $1.26(0.11)$ & $2.16(0.10)$ & 0.918 \\
\hline $\begin{array}{l}\text { Mean interval between hexavalent vaccine dose } \\
3 \text { and blood sample month } 9 \text { in months (SEM) }\end{array}$ & $4.42(0.10)$ & $4.39(0.30)$ & 0.984 \\
\hline $\begin{array}{l}\text { Mean interval between hexavalent vaccine dose } \\
3 \text { and blood sample month } 12 \text { in months (SEM) }\end{array}$ & $7.42(0.07)$ & $7.82(0.13)$ & 0.007 \\
\hline
\end{tabular}

Table 1: Demographic characteristics of the participating women and infants. Statistical test used: unpaired t-test and chi-square test. 


\begin{tabular}{|c|c|c|c|c|c|c|}
\hline $\begin{array}{l}\text { GMC }(95 \% \\
\text { CI) in women }\end{array}$ & \multicolumn{2}{|c|}{ Anti-FHA (EU/mL) } & \multicolumn{2}{|c|}{ Anti-Prn (EU/mL) } & \multicolumn{2}{|c|}{ Anti-PT (EU/mL) } \\
\hline $\begin{array}{l}\text { DELIVERY } \\
\text { CHILD } 1\end{array}$ & \multicolumn{2}{|l|}{$\begin{array}{l}21.50 \\
(16.96-27.22)\end{array}$} & \multicolumn{2}{|l|}{$\begin{array}{l}15.84 \\
(11.97-20.96)\end{array}$} & \multicolumn{2}{|l|}{$\begin{array}{l}6.47 \\
(5.16-8.11)\end{array}$} \\
\hline N samples & \multicolumn{2}{|l|}{84} & \multicolumn{2}{|l|}{85} & \multicolumn{2}{|l|}{85} \\
\hline $\begin{array}{l}\text { ONE MONTH } \\
\text { AFTER } \\
\text { VACCINATION }\end{array}$ & \multicolumn{2}{|c|}{$\begin{array}{l}770.75 \\
(638.43-930.49)\end{array}$} & \multicolumn{2}{|l|}{$\begin{array}{l}658.88 \\
(503.92-861.49)\end{array}$} & \multicolumn{2}{|l|}{$\begin{array}{l}69.97 \\
(55.87-87.62)\end{array}$} \\
\hline N samples & \multicolumn{2}{|l|}{86} & \multicolumn{2}{|l|}{86} & \multicolumn{2}{|l|}{86} \\
\hline $\begin{array}{l}\text { DELIVERY } \\
\text { CHILD } 2 \\
\end{array}$ & \multicolumn{2}{|c|}{$\begin{array}{l}149.59 \\
(118.22-189.28)\end{array}$} & \multicolumn{2}{|c|}{$\begin{array}{l}147.91 \\
(100.37-217.96)\end{array}$} & \multicolumn{2}{|l|}{$\begin{array}{l}13.43 \\
(10.10-17.85)\end{array}$} \\
\hline N samples & \multicolumn{2}{|l|}{55} & \multicolumn{2}{|l|}{55} & \multicolumn{2}{|l|}{55} \\
\hline \multirow{2}{*}{$\begin{array}{l}\text { GMC (95\% } \\
\text { CI) in infants }\end{array}$} & \multicolumn{2}{|c|}{ Anti-FHA (EU/mL) } & \multicolumn{2}{|c|}{ Anti-Prn (EU/mL) } & \multicolumn{2}{|c|}{ Anti-PT (EU/mL) } \\
\hline & GROUP A & GROUP B & GROUP A & GROUP B & GROUP A & GROUP B \\
\hline CORD & $\begin{array}{l}32.16 \\
(22.49-45.99)\end{array}$ & $\begin{array}{l}239.85 \\
(187.93- \\
306.12)\end{array}$ & $\begin{array}{l}22.60 \\
(15.66-32.63)\end{array}$ & $\begin{array}{l}253.74 \\
(175.35-367.17)\end{array}$ & $\begin{array}{l}9.68 \\
(6.86-13.65)\end{array}$ & $\begin{array}{l}22.61 \\
(17.18-29.74)\end{array}$ \\
\hline p-value & \multicolumn{2}{|c|}{$<0.001$} & \multicolumn{2}{|r|}{$<0.001$} & \multicolumn{2}{|c|}{$<0.001$} \\
\hline N samples & 46 & 55 & 48 & 55 & 48 & 55 \\
\hline MONTH 1 & $\begin{array}{l}16.70 \\
(10.84-25.73)\end{array}$ & $\begin{array}{l}133.63 \\
(105.38- \\
169.44)\end{array}$ & $\begin{array}{l}10.89 \\
(7.04-16.86)\end{array}$ & $\begin{array}{l}143.49 \\
(101.31-203.25)\end{array}$ & $\begin{array}{l}5.46 \\
(3.91-7.63)\end{array}$ & $\begin{array}{l}11.84 \\
(8.81-15.92)\end{array}$ \\
\hline p-value & \multicolumn{2}{|c|}{$<0.001$} & \multicolumn{2}{|r|}{$<0.001$} & \multicolumn{2}{|c|}{0.001} \\
\hline N samples & 34 & 53 & 35 & 53 & 35 & 53 \\
\hline MONTH 6 & $\begin{array}{l}206.42 \\
(116.36- \\
366.17) \\
\end{array}$ & $\begin{array}{l}92.40 \\
(67.79- \\
125.96) \\
\end{array}$ & $\begin{array}{l}70.34 \\
(41.63-118.85)\end{array}$ & $\begin{array}{l}25.27 \\
(14.45-44.18)\end{array}$ & $\begin{array}{l}59.63 \\
(34.25-103.83)\end{array}$ & $\begin{array}{l}36.31 \\
(27.17-48.53)\end{array}$ \\
\hline p-value & \multicolumn{2}{|c|}{0.039} & \multicolumn{2}{|r|}{0.016} & \multicolumn{2}{|c|}{0.175} \\
\hline N samples & 15 & 11 & 15 & 11 & 15 & 11 \\
\hline MONTH 9 & $\begin{array}{l}84.28 \\
(64.82- \\
109.57) \\
\end{array}$ & $\begin{array}{l}32.58 \\
(22.19-47.85)\end{array}$ & $\begin{array}{l}36.89 \\
(25.72-52.90)\end{array}$ & $\begin{array}{l}12.19 \\
(6.97-21.33)\end{array}$ & $\begin{array}{l}23.76 \\
(17.33-32.58)\end{array}$ & $\begin{array}{l}16.23 \\
(11.29-23.31)\end{array}$ \\
\hline p-value & & & & 0.002 & & .154 \\
\hline N samples & 20 & 10 & 19 & 10 & 19 & 10 \\
\hline MONTH 12 & $\begin{array}{l}57.75 \\
(45.28-73.65)\end{array}$ & $\begin{array}{l}27.99 \\
(21.02-37.28)\end{array}$ & $\begin{array}{l}12.31 \\
(8.79-17.24)\end{array}$ & $\begin{array}{l}7.05 \\
(5.52-9.01)\end{array}$ & $\begin{array}{l}10.87 \\
(8.50-13.90)\end{array}$ & $\begin{array}{l}6.39 \\
(5.07-8.04)\end{array}$ \\
\hline p-value & & & & 0.009 & & .003 \\
\hline $\mathrm{N}$ samples & 33 & 47 & 32 & 47 & 32 & 47 \\
\hline
\end{tabular}

Table 2: Geometric Mean Concentration (GMC) with 95\% confidence interval (95\% CI) for antibody concentrations against Filamentous Hemagglutinin (FHA), Pertactin (Prn) and Pertussis Toxin (PT) in women and infants at different time points, expressed in Elisa Units per milliliter (EU/mL).There was not enough sample available anymore from all women at the delivery of the first born child. Statistical test used: unpaired t-test. 


\begin{tabular}{|l|l|l|l|}
\hline Mean ratio (SEM) & \multicolumn{2}{|c|}{ Transplacental transport ratio } & p-value \\
\hline & Group A infants & Group B infants & \\
\hline Pertussis Toxin IgG & $1.96(0.18)$ & $1.76(0.08)$ & 0.300 \\
\hline $\begin{array}{l}\text { Filamentous Hemagglutinin } \\
\text { IgG }\end{array}$ & $1.78(0.09)$ & $1.74(0.07)$ & 0.899 \\
\hline Pertactin IgG & & & 0.147 \\
\hline
\end{tabular}

Table 3: Transplacental transport ratio for Pertussis Toxin, Filamentous haemagglutinin and pertactin antibodies in group A and group B infants. Statistical test used: unpaired t-test. 


\section{Effect of Pre-pregnancy Pertussis Vaccination in Young Infants Appendix}

\section{Model building for data in infants:}

Dynamics of antibodies in infants:

There are maximum four data points of anti-PT IgG antibody in each infant: at delivery (cord blood sample), 1 month after birth, 6 or 9 months after birth and 12 months after birth. Based on the recommended vaccination schedule in Belgium, infants were vaccinated at the ages of 8,12 and 16 weeks. We propose to use the model with three ODEs (see Figure 1).

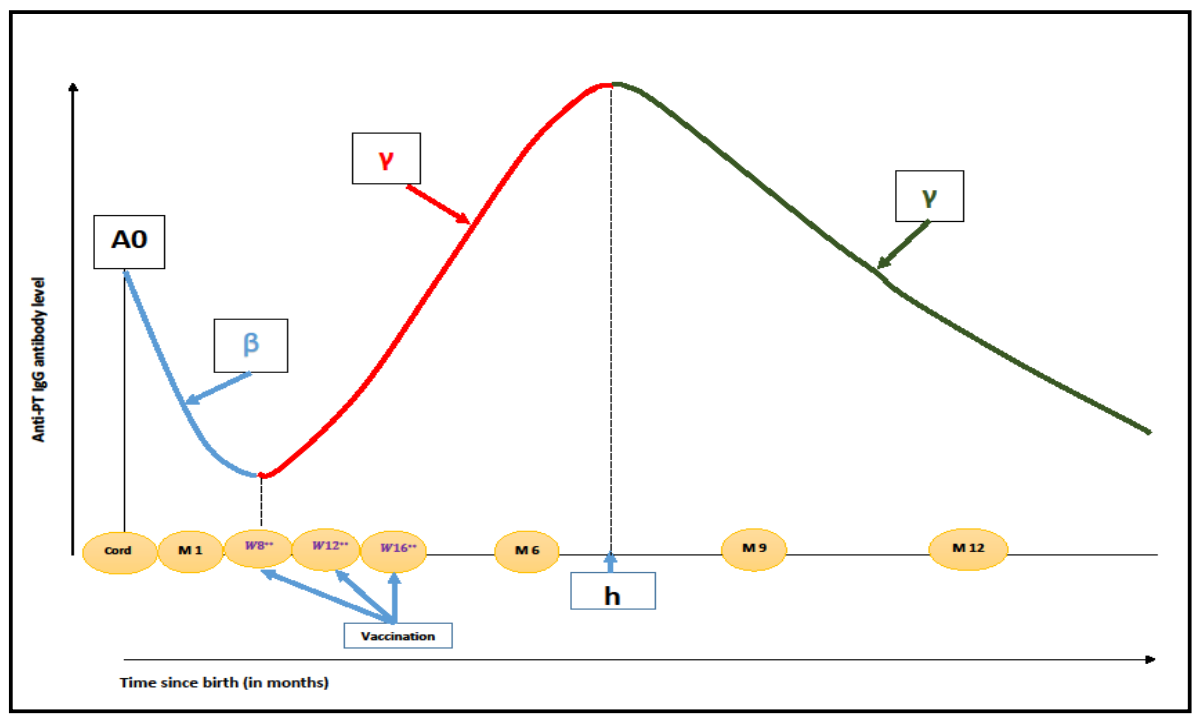

Figure 1: Illustrated presumed dynamics of anti-pertussis antibody levels in infants: $A_{0}$ is the antibody level at birth. After birth, the antibody levels decrease with decay rate $\beta$. After the administration of the first aP containing vaccine at week 8, the antibodies start to increase up to time point $h$ at an overall rate $\gamma$. It is hypothesized that $h>4$ since the infants are vaccinated at week 8,12 and 16 . After time point $h$, antibodies decrease again at rate $\alpha$. Cord, M1, M6, M9 and M12 indicate the sampling time points. $W 8^{* *}, W 12^{* *}$ and $W 16^{* *}$ indicate the vaccination time points.

The dynamic change in anti-pertussis antibodies is written down as follows:

$$
\begin{gathered}
t \leq 2: \frac{d A}{d t}=-\beta A \\
2<t \leq h: \frac{d A}{d t}=\gamma A \\
t>h: \frac{d A}{d t}=-\alpha A
\end{gathered}
$$


This ODE system assumes that anti-PT IgG antibodies firstly decrease with a decay $\beta$ right after birth till the $1^{\text {st }}$ vaccination at month two. After the $1^{\text {st }}$ vaccination, antibodies increase with an overall rate of $\gamma$ which is accounted for the general increase due to three consecutive vaccination doses. Since there are not enough data points to model the increasing rate after each vaccination dose, only the overall rate $\gamma$ is expressed and modelled. After the vaccination at month four, antibodies remain increasing, till time point $h$, and start decrease again with decay $\alpha$.

\section{Model building and selection:}

Model selection is performed based on AIC obtained from models without any covariate fit in Monolix software. The anti-PT IgG antibody level at $T_{0}$ (corresponding to cord sample) is $A_{0}$. Since there are maximum four measurements per subjects, we allow maximum four random effects. We let $h$ fixed, and let $A_{0, i}, \beta_{i}, \gamma_{i}$ and $\alpha_{i}$ be random. The individual parameters are assumed to have log-normal distribution.

To perform model selection, we fit different models with several scenarios: models with only 1 random effect, models with two random effects, models with three random effects and model with four random effects. Some models did not achieve convergence in Monolix software. Among all models whose convergence was achieved, the model with four random effects had the lowest AIC. As a result, we used this model to continue our analysis, where covariates were taken into account.

First of all, we consider the order of the infants (covariate child2: child2 $=0$ if the child from group A infants and child $2=1$ if the child from group B siblings) as a categorical variable with group A infants as reference group . The model where child2 is assumed to affect the estimate of all parameters did not converge in Monolix. The results presented in the manuscript, hence, were obtained from the model where child2 was assumed to affect the estimate of four parameters $\left(A_{0, i}, \beta_{i}, \gamma_{i}, \alpha_{i}\right)$. Directly after, the effect of gender (covariate gender: gender $=1$ if male and gender $=0$ if female where baby girl is the reference group), birthweight (covariate bweight centered around its mean bweight_mean) of infants (in gram) were additionally taken into account.

\section{Specification for final models:}

- $\quad$ Model with only one covariate (child2):

Let denote $A_{0}, \beta, \gamma$ and $\alpha$ the four population parameters. Individual parameters are assumed to have log-normal distribution, it follows:

$$
\begin{gathered}
\log \left(A_{0, i}\right)=\log \left(A_{0}\right)+\rho_{A_{0}} \operatorname{child} 2_{i}+\eta_{A_{0, i}} \\
\log \left(\beta_{i}\right)=\log (\beta)+\rho_{\beta} \operatorname{child} 2_{i}+\eta_{\beta, i} \\
\log \left(\gamma_{i}\right)=\log (\gamma)+\rho_{\gamma} \operatorname{child} 2_{i}+\eta_{\gamma, i} \\
\log \left(\alpha_{i}\right)=\log (\alpha)+\rho_{\alpha} \operatorname{child} 2_{i}+\eta_{\alpha, i}
\end{gathered}
$$

Where:

$$
\begin{aligned}
\eta_{A_{0, i}} & \sim N\left(0, \delta_{A_{0}}{ }^{2}\right), \\
\eta_{\beta, i} & \sim N\left(0, \delta_{\beta}{ }^{2}\right),
\end{aligned}
$$




$$
\begin{aligned}
\eta_{\gamma, i} & \sim N\left(0, \delta_{\gamma}{ }^{2}\right) \\
\eta_{\alpha_{i}} & \sim N\left(0, \delta_{\alpha}{ }^{2}\right),
\end{aligned}
$$

We use anti-PT IgG antibody levels on the $\log 10$ scale and assume an additive residual error model, that is:

$$
\log _{10}\left(A_{o b s, i j}\right)=\log _{10}\left(A_{\text {pred }, i j}\right)+\varepsilon_{i j}
$$

where $A_{o b s, i j}$ and $A_{p r e d, i j}$ are the observed and predicted anti-PT IgG antibodies for the child $i$ at time point $j$. The residual error is assumed to have a normal distribution with mean 0 , that is:

$$
\varepsilon_{i j} \sim N\left(0, \delta^{2}\right),
$$

- Model with three covariates (child2, gender, bweigth):

Initially, we fit the models where only gender or bweight was considered as covariate separately. In the next step, only statistically significant relationship of gender and bweight was retained in the final model with three covariates since the central inference lies on the effect of child2.

The individual parameters in the final model were assumed to have log-normal distribution:

$$
\begin{aligned}
& \log \left(A_{0, i}\right)=\log \left(A_{0}\right)+\rho_{\text {child } 2, A_{0}}{\text { child } 2_{i}}+\rho_{\text {gender }, A_{0}} \text { gender }_{i}+\eta_{A_{0, i}} \\
& \log \left(\beta_{i}\right)=\log (\beta)+\rho_{\text {child } 2, \beta}{\text { child } 2_{i}}+\rho_{\text {bweight }, \beta}\left(\text { bweight }_{i}-\text { bweight_mean }\right)+\eta_{\beta, i} \\
& \log \left(\gamma_{i}\right)=\log (\gamma)+\rho_{\text {child } 2, \gamma} \operatorname{child} 2_{i}+\eta_{\gamma, i} \\
& \log \left(\alpha_{i}\right)=\log (\alpha)+\rho_{\text {child }, \alpha}{\text { child } 2_{i}}+\rho_{\text {gender }, \alpha} \text { gender }_{i}+\eta_{\alpha, i}
\end{aligned}
$$

Moreover, gender is assumed to affect the estimate of $h$.

- Left-censored observations:

There are anti-PT IgG antibody values less than the limit of detection ( $5 \mathrm{IU} / \mathrm{mL}$ ). These data will be treated as left censored during the estimation procedure in Monolix software.

\section{Model diagnosis:}

We perform model diagnosis for the final model by the means of the SAEM (Stochastic Approximation Expectation Maximization) convergence plot and residual plots (residuals v.s time and residuals v.sprediction).

Furthermore, the scatter plot of the observed antibody levels and predicted values are presented to partially evaluate the prediction ability of the model (see Figure 2). 


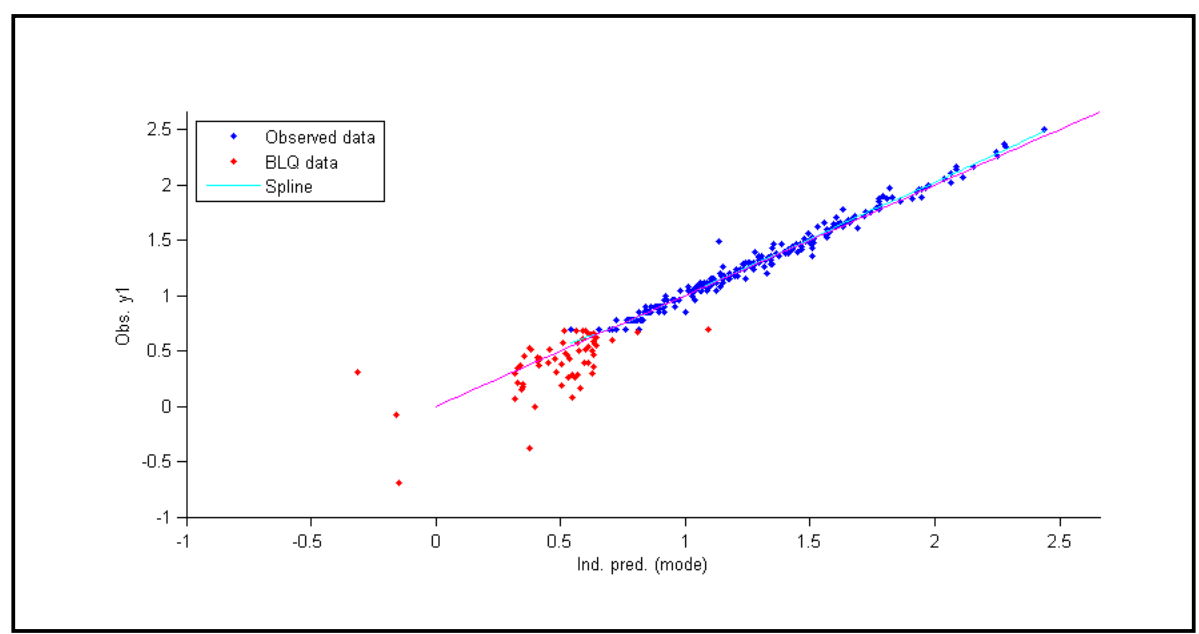

Figure 2: Scatter plot of the observed anti-PT IgG antibodies with respect to the predicted anti-PT IgG antibodies using the individual parameters (model in infants with only one covariate child2, output from Monolixsoftware).

\section{Important results:}

In the model with three covariates, it is shown that boys were born with significantly higher anti-PT IgG concentrations compared to girls in both groups of infants. The median anti-PT IgG antibody levels at birth in group A infants is respectively $8.51 \mathrm{EU} / \mathrm{mL}$ (IQR: 5.96 - 11.06) for girls and 13.3 $\mathrm{EU} / \mathrm{mL}$ (IQR: 9.18 - 17.42) for boys; and in group B siblings $18.20 \mathrm{EU} / \mathrm{mL}$ (IQR: 17.63 - 18.77) for girls and $28.40 \mathrm{EU} / \mathrm{mL}$ (IQR: 27.55 - 29.25) for boys,respectively. 


\section{Model building for data in pregnant women:}

\section{Dynamics of antibodies in pregnant women:}

There were three blood sampling occasions in each pregnant woman: at the $1^{\text {st }}$ delivery, 1 month after booster vaccination and at the delivery of the $2^{\text {nd }}$ child.

We denoted the antibody level at $T_{0}\left(1^{\text {st }}\right.$ delivery $)$ as $A_{0}$. In theory, antibody levels decrease over time until the women received a booster vaccination. From that moment, antibodies are assumed to immediately increase. Since we do not have enough data to observe the whole process, what we can model was the process illustrated in Figure 3. In this presumed dynamics, the solid line (in black) illustrates the theoretical change of antibodies. The dashed-dotted line (in red) is the curve based on the collected data. It is assumed that the decay rates after the peak (time point $h$ ) were the same in solid line and dashed line. However, it is a strong assumption and the conclusion based on the result of $\alpha$ should be interpreted with caution.

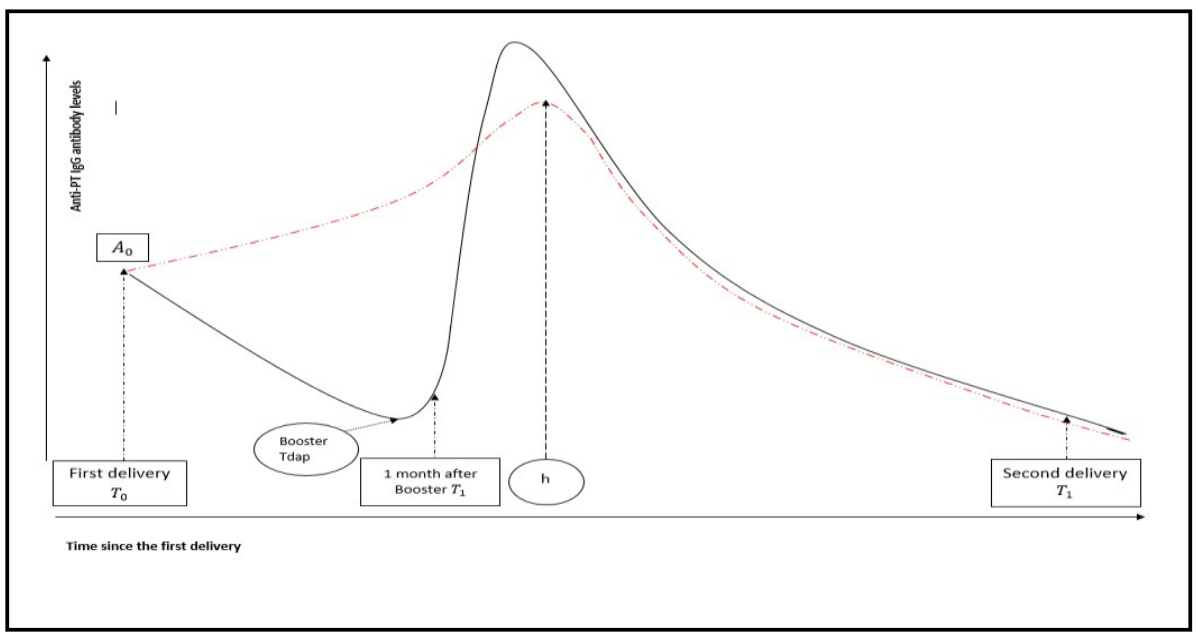

Figure 3: Illustrated presumed dynamics of antibody levels in pregnant women. Solid line (in black): The theoretical dynamics of antibodies, where it is assumed to decrease over time and then immediately increase after pregnant women received the booster vaccination. Dashed-dotted line (in red): The hypothesized dynamics of antibodies based on the collected data where it is assumed that antibodies increase between $T_{0}$ and $T_{1}$ with the rate $\omega$ then decrease between $T_{1}$ and $T_{2}$ with the decay $\alpha$.

The ODEs for the evolution of antibodies in pregnant women are written as follows:

$$
\begin{aligned}
& t \leq h: \frac{d A}{d t}=\omega A, \\
& t>h: \frac{d A}{d t}=-\alpha A .
\end{aligned}
$$

It is noticed that some notations used in this section, though might be the same as those in the infants' model, in fact carry their own meaning and values.

\section{Model building and selection:}

We fit non-linear mixed effects model using Monolix software. Model selection was performed based on AIC (smaller is better). Since there were maximum three measurements per subjects, we allowed maximum three random effects. We let $h$ fixed, and let $A_{0}$, $\omega$ and $\alpha$ be random (model A). This mode , however, did not converge in Monolix. Based on the SAEM convergence plot, there seemed to be a negative correlation between $h$ and $\omega$. To further explore this notice, we extracted the sampled values of $h$ and $\omega$ during SAEM in Monolix. The scatter plot (in Figure 4) shows a curvature relationship between the two variables. 


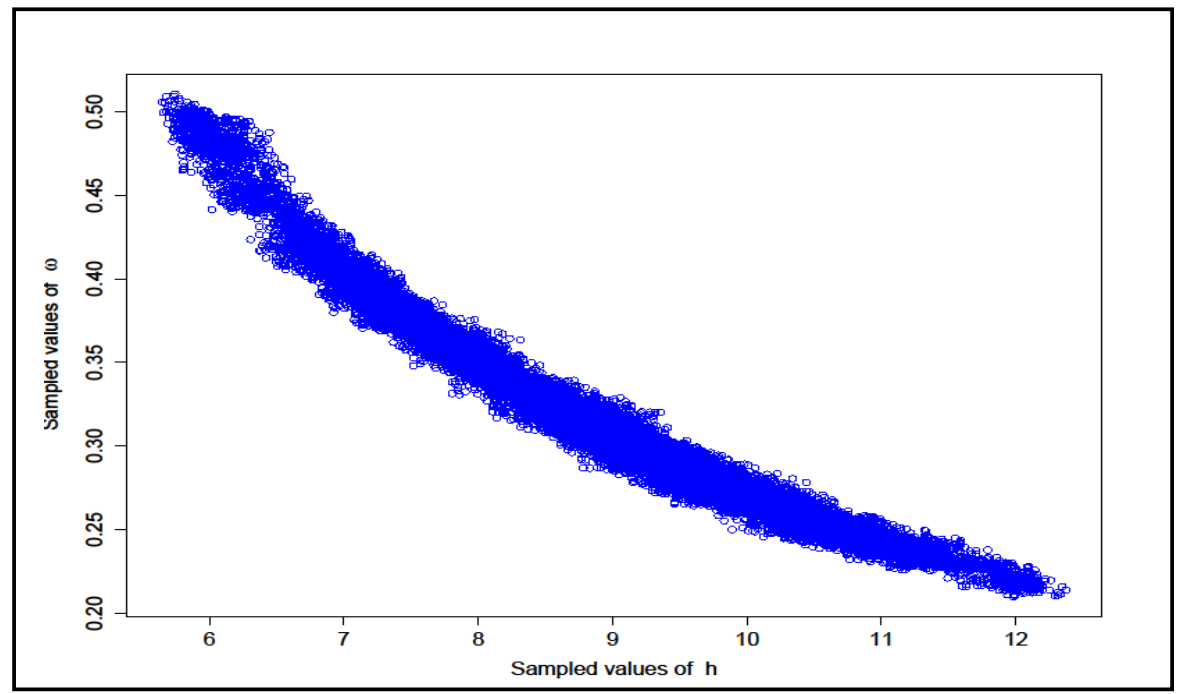

Figure 4: The scatter plot between sampled values of $h$ and sampled values of $\omega$ from running model A in Monolix software.

We then applied the transformation to stabilize the sampling procedure in Monolix: $\omega=\frac{h}{e^{\gamma}}$ As a result, instead of estimating $\omega$ directly, we estimate $\gamma$.

The four-parameter model $\left(A_{0}, \gamma, \alpha\right.$, and $\left.h\right)$ with three random effects $\left(A_{0, i}, \gamma_{i}, \alpha_{i}\right)$ were fit. Based on the SAEM convergence plot produced from Monolix software, the convergence of $\gamma$ seems to be very unstable. Hence, a two-random-effect model $\left(A_{0, i}, \alpha_{i}\right)$ was fit. The convergence of this model was well achieved.

To perform model selection, we run two models with only 1 random effect: $A_{0, i}$ or $\alpha_{i}$ separately. The AIC obtained from these two models are higher compared to the AIC of model with two random effects $\left(A_{0, i}, \alpha_{i}\right)$. As a result, the final model is the model with two random effects $\left(A_{0, i}, \alpha_{i}\right)$.

Specification for final model:

Let denote $A_{0}$ and $\alpha$ the two population parameters. The age at boostrix (centered around its mean: age_mean) is assumed to affect the estimate of all four parameters. We assume $A_{0, i}$ and $\alpha_{i}$ have lognormal distribution:

$$
\begin{gathered}
\log \left(A_{0, i}\right)=\log \left(A_{0}\right)+\beta_{A_{0}}\left(\text { age }_{i}-\text { age_mean }\right)+\eta_{A_{0, i}} \\
\log \left(\alpha_{i}\right)=\log (\alpha)+\beta_{\alpha}\left(\text { age } e_{i}-\text { age_mean }\right)+\eta_{\alpha, i}
\end{gathered}
$$

Where

$$
\begin{aligned}
\eta_{A_{0, i}} & \sim N\left(0, \delta_{A_{0}}{ }^{2}\right), \\
\eta_{\alpha_{i}} & \sim N\left(0, \delta_{\alpha}{ }^{2}\right),
\end{aligned}
$$

We use anti-PT IgG antibody levels on the log10 scale and assume an additive residual error model, that is:

$$
\log _{10}\left(A_{o b s, i j}\right)=\log _{10}\left(A_{\text {pred }, i j}\right)+\varepsilon_{i j}
$$

where $A_{o b s, i j}$ and $A_{\text {pred, } i j}$ are the observed and predicted anti-PT IgG antibodies for women $i$ at time $j$. The residual error is assumed to have a normal distribution with mean 0 , that is:

$$
\varepsilon_{i j} \sim N\left(0, \delta^{2}\right),
$$

There are antibody values less than the limit of detection $(5 \mathrm{IU} / \mathrm{mL})$. These data will be treated as left censored during the estimation procedure in Monolix software.

Model diagnosis:

We perform model diagnosis for the final model by the means of the SAEM convergence plot and residual plots (residuals v.s time and residuals v.s prediction). 


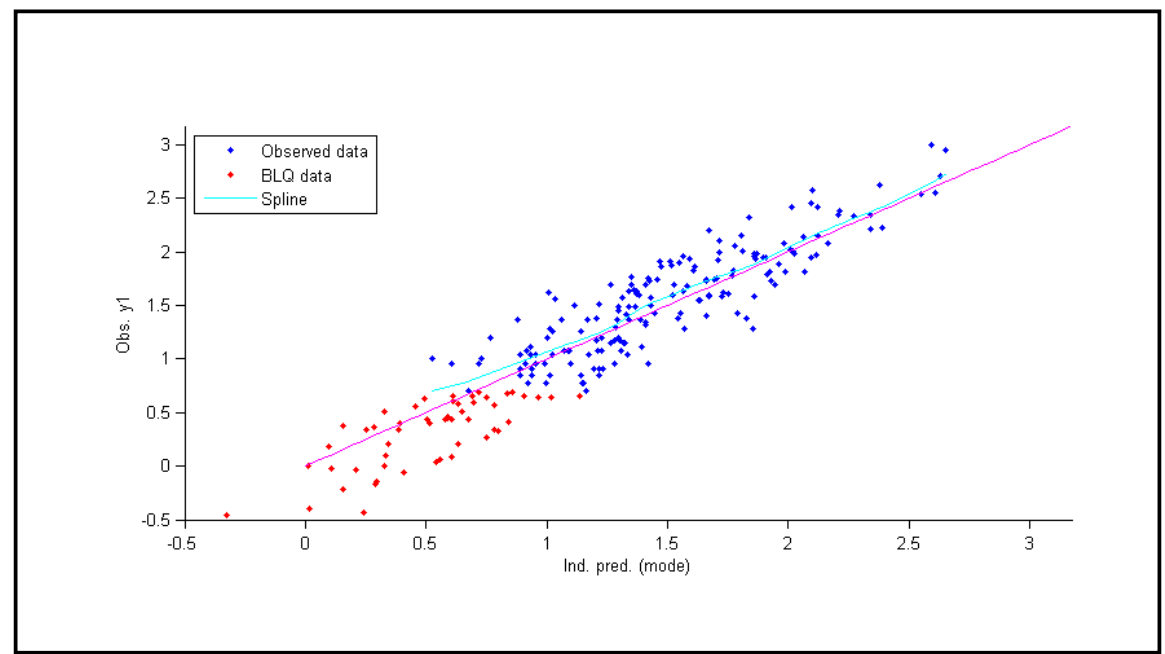

Figure 5: Scatter plot of the observed anti-PT IgG antibody levels with respect to the predicted antiPT IgG antibody levels using the individual parameters (model in pregnant women, output from Monolix software). 


\section{Robust linear regression model to investigate the association between the anti-PT IgG antibodies in pregnant women at delivery and that in Cords of their infants:}

Figure 6 shows the scatter plot between anti-PT IgG antibodies in Cords of infants and that in pregnant women at delivery. It is expected to see a linear trend between the two measurements.

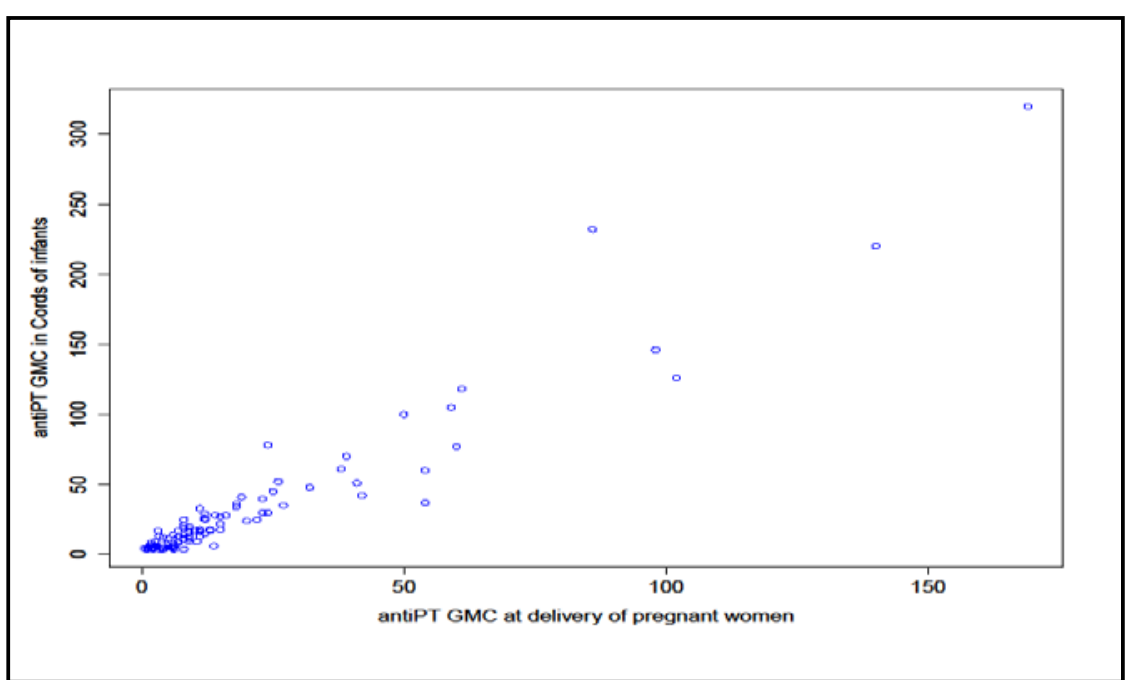

Figure 6: Scatter plot between anti-PT IgG antibodies in Cords of infants and that in pregnant women at delivery. Left censored observations were replaced by the individual predicted mode values obtained from the final NLMM models for data in infants and data in pregnant women.

Model building and selection:

The response variable is antiCord (anti-PT IgG antibodies in Cords of infants). Initially, a full model with four covariates: anti-PT IgG antibodies of pregnant women at delivery (antiPTMum covariate), the order of the child (child2), the gender (gender) and birthweight of the child (bweight covariate) along with the interaction between child2 and antiPTMum was considered. However, this model did not satisify two important assumptions, namely, the normality and the homocesdasticity of the residuals. Hence, it is decided to transform the response variable and the antiPTMum covariate into natural $\log$ scale. The motivation to choose this transformation is that it helps to satisfy the assumptions of linear model, and among other potential transformations, it serves the purpose of easier interpretation. The results from model on transformed data shown that the effects of gender, bweight, child 2 and the interaction between antiPTMum and child2 were not significant. Since the central interest lies in the association between the antibody levles in pregnant women at delivery and that in Cords of their infants, the final model is the simple linear regression model where only antiPTMum is considered ascovariate.

Specification for final model:

The final model could be written down as follows:

$$
\log \left(\operatorname{antiCord}_{i}\right)=\log \left(\operatorname{antiPTMum}_{i}\right)+\varepsilon_{i},
$$

where $i=1,2, \ldots, 97$.

The assumption of homocesdasticity of the residuals is not satisfied. Hence, a robust linear regression model was fit using $r l m$ function in the MASS package. The result from this robust linear regression was used for making inference. 